\title{
RNA sequencing (RNA-Seq) of lymph node, spleen, and thymus transcriptome from wild Peninsular Malaysian cynomolgus macaque (Macaca fascicularis)
}

\begin{abstract}
Joey Ee Uli ${ }^{1}$ ， Christina Seok Yien Yong ${ }^{2}$ ， Swee Keong Yeap ${ }^{3}$ ， Jeffrine J. Rovie-Ryan ${ }^{4}$ ， Nurulfiza Mat Isa ${ }^{1}$, Soon Guan Tan ${ }^{1}$, Noorjahan Banu Alitheen ${ }^{\text {Corresp. } 1}$

1 Department of Cell and Molecular Biology, Faculty of Biotechnology and Biomolecular Sciences, Universiti Putra Malaysia, Serdang, Selangor, Malaysia

3 China-ASEAN College of Marine Sciences, Xiamen University, Sepang, Selangor, Malaysia

4 Department of Wildlife and National Parks (DWNP), Ex-Situ Conservation Division, Department of Wildlife and National Parks, Kuala Lumpur, Malaysia
\end{abstract} Corresponding Author: Noorjahan Banu Alitheen

Email address: noorjahan@upm.edu.my

The cynomolgus macaque (Macaca fascicularis) is an extensively utilised nonhuman primate model for biomedical research due to its biological, behavioural, and genetic similarities to humans. Genomic information of cynomolgus macaque is vital for research in various fields, however, there is presently a shortage of genomic information on the Malaysian cynomolgus macaque. This study aimed to sequence, assemble, annotate, and profile the Peninsular Malaysian cynomolgus macaque transcriptome derived from three tissues, lymph node, spleen, and thymus using RNA sequencing (RNA-Seq) technology. A total of $174,208,078$ paired end 70 base pair sequencing reads were obtained from the Illumina Hi-Seq 2500 sequencer. The overall mapping percentage of the sequencing reads to the $M$. fascicularis reference genome ranged from $53-63 \%$. Categorisation of expressed genes to Gene Ontology (GO) and KEGG pathway categories revealed that GO terms with the highest number of associated expressed genes include Cellular process, Catalytic activity, and Cell part, while for pathway categorisation, the majority of expressed genes in lymph node, spleen, and thymus fall under the Global overview and maps pathway category, while 266, 221, and 138 genes from lymph node, spleen, and thymus were respectively enriched in the Immune system category. Enriched Immune system pathways include Platelet activation pathway, Antigen processing and presentation, B cell receptor signalling pathway, and Intestinal immune network for IgA production. Differential gene expression analysis among the three tissues revealed 574 differentially expressed genes (DEG) between lymph and spleen, 5402 DEGs between lymph and thymus, and 7008 DEGs between spleen and thymus. Venn diagram analysis of expressed genes revealed a total of 2630, 253, and 279 tissue-specific genes respectively for lymph node, spleen, and thymus tissues. This is the first time the lymph node, spleen, and thymus transcriptome of the 
Peninsular Malaysian cynomolgus macaque have been sequenced via RNA-Seq. Novel transcriptomic data will further enrich the present $M$. fascicularis genomic database and provide future research potentials, including novel transcript discovery, comparative studies, and molecular markers development. 
1 RNA sequencing (RNA-Seq) of lymph node, spleen, and thymus transcriptome from wild

2 Peninsular Malaysian cynomolgus macaque (Macaca fascicularis).

3

4 Joey Ee Uli ${ }^{1}$, Christina Seok Yien Yong ${ }^{2}$, Swee Keong Yeap ${ }^{3}$, Jeffrine J. Rovie-Ryan ${ }^{4}$, Nurulfiza

5 Mat Isa $^{1}$, Soon Guan Tan ${ }^{1}$ and Noorjahan Banu Alitheen ${ }^{1}$

$6{ }^{1}$ Department of Cell and Molecular Biology, Faculty of Biotechnology and Biomolecular

7 Sciences, Universiti Putra Malaysia, 43400 Serdang, Selangor, Malaysia

8 2Department of Biology, Faculty of Science, Universiti Putra Malaysia, 43400 Serdang,

9 Selangor, Malaysia

${ }^{3}$ China-ASEAN College of Marine Sciences, Xiamen University Malaysia, Jalan Sunsuria, Bandar Sunsuria, 43900 Sepang, Selangor, Malaysia

${ }^{4}$ Wildlife Genetic Resource Bank (WGRB) Laboratory, Ex-Situ Conservation Division, Department of Wildlife and National Parks (DWNP) Peninsular Malaysia, KM 10 Cheras Road, 56100 Kuala Lumpur, Malaysia

Corresponding Author:

Noorjahan Banu Alitheen

Email: noorjahan@upm.edu.my 
Abstract

The cynomolgus macaque (Macaca fascicularis) is an extensively utilised nonhuman primate model for biomedical research due to its biological, behavioural, and genetic similarities to humans. Genomic information of cynomolgus macaque is vital for research in various fields, however, there is presently a shortage of genomic information on the Malaysian cynomolgus macaque. This study aimed to sequence, assemble, annotate, and profile the Peninsular Malaysian cynomolgus macaque transcriptome derived from three tissues, lymph node, spleen, and thymus using RNA sequencing (RNA-Seq) technology. A total of 174,208,078 paired end 70 base pair sequencing reads were obtained from the Illumina Hi-Seq 2500 sequencer. The overall mapping percentage of the sequencing reads to the $M$. fascicularis reference genome ranged from 53-63\%. Categorisation of expressed genes to Gene Ontology (GO) and KEGG pathway categories revealed that GO terms with the highest number of associated expressed genes include Cellular process, Catalytic activity, and Cell part, while for pathway categorisation, the majority of expressed genes in lymph node, spleen, and thymus fall under the Global overview and maps pathway category, while 266, 221, and 138 genes from lymph node, spleen, and thymus were respectively enriched in the Immune system category. Enriched Immune system pathways include Platelet activation pathway, Antigen processing and presentation, B cell receptor signalling pathway, and Intestinal immune network for IgA production. Differential gene expression analysis among the three tissues revealed 574 differentially expressed genes (DEG) between lymph and spleen, 5402 DEGs between lymph and thymus, and 7008 DEGs between spleen and thymus. Venn diagram analysis of expressed genes revealed a total of 2630, 253, and 279 tissue-specific genes respectively for lymph node, spleen, and thymus tissues. This is the first time the lymph node, spleen, and thymus transcriptome of the Peninsular Malaysian cynomolgus macaque have been sequenced via RNA-Seq. Novel transcriptomic data will further enrich the present $M$. fascicularis genomic database and provide future research potentials, including novel transcript discovery, comparative studies, and molecular markers development.

Introduction

The cynomolgus macaque (Macaca fascicularis), also known as the long-tailed macaque or crab-eating macaque, are nonhuman primates (NHP) belonging to the Cercopithecidae family. They occur in the southeastern region of Bangladesh, the mainland of Southeast Asia (including Malaysia, Laos, Cambodia, Vietnam, and Thailand), the islands of Indonesia, Borneo, and the Philippines and the Nicobar Islands of India (Groves, 2001), and more recently Mauritius (Ferguson et al., 2007). Out of the 50 Macaca fascicularis subspecies presently classified, the Macaca fascicularis fascicularis subspecies is the most widely distributed macaque subspecies 
in Peninsular Malaysia (Abdul-Latiff et al., 2014), mostly occurring in mangrove forests, lowland rainforests, and in the peripheral of urban dwellings.

33
evolutionary relationship with humans (Perelman et al., 2011). Biological, physiological, behavioural, and genetic similarities between humans and cynomolgus macaques make these macaques capable of recapitulating symptoms of diseases observed in humans (Patel, Jhamb \& Singh, 2011; Shively et al., 2015). As a result, the cynomolgus macaques are regarded as suitable model organisms for translational studies in the biomedical field (Carlsson et al., 2004). Their inclusion in numerous immunological, neuroscience, vaccine development, and pharmacokinetic studies have shown the cynomolgus macaque's versatility as NHP model organisms (Higgs \& Ziegler, 2010; Nunamaker et al., 2013; Lee et al., 2014b; Berry et al., 2015). In order to benefit the biomedical field, complete genomic and transcriptomic information has become essential in expediting the understanding of gene expressions and biological pathways relevant to studies of interest. Asides from biomedicine, complete genomic and transcriptomic information enables researchers to perform phylogenomic studies of relevant organisms. Aligning entire genomes enables a higher base resolution to infer phylogeny. High throughput sequencing serves as a tool to obtain genomic and transcriptomic information of any organism of interest from relatively small starting material and at a fraction of the cost involved in Sanger and capillary shotgun sequencing approaches. In recent years, RNA sequencing (RNA-Seq) has become an indispensable method to sequence entire transcriptomes. Downstream applications of RNA-Seq include differential gene expression analyses, expression profiling, novel transcript discovery, single nucleotide polymorphism (SNP) discovery, and also development of molecular markers for population genetics studies (Han et al., 2015). the cynomolgus macaque from various locations using the high-throughput sequencing approach (Yan et al., 2011; Higashino et al., 2012; Osadaet al., 2015). To date, the transcriptome of the Malaysian cynomolgus macaque has yet to be sequenced, and while the transcriptomes of the lymph node, spleen, and thymus tissues harvested from cynomolgus macaques have already been sequenced via various high-throughput sequencing platforms, the individuals sequenced were of Mauritian, Vietnamese, Chinese, and Philippine origin (Ebeling et al., 2011; Huh et al., 2012; Lee et al., 2014a; Peng et al., 2014). In addition, to the best of our knowledge, the macaque transcriptomes sequenced in these previous studies were individuals raised in laboratory conditions. While it is beneficial for biomedical research that model organisms like cynomolgus macaques are bred in controlled laboratory conditions to minimise genetic and phenotypic variations, it ultimately does not reflect the variable environment in which humans live in (Pedersen \& Babayan, 2011). Comparisons between wild and laboratory strains of Mus musculus have shown the wild strain to possess more variable immune responses compared with the laboratory strain (Abolins et al., 2011). 
113

114

115

116

117

118

119

120

121

122

123

124

125

126

127

128

129

130

131

132

133

134

135

136

137

138

139

140

141

142

143

144

145

146

147

148

149

Furthermore, genetic variations among different populations of NHP models have resulted in varying phenotypical reactions to treatments in biomedical research. One such observation was made in the Indian and Chinese rhesus macaques (Macaca mulatta), whereby Chinese rhesus macaques exhibited slower progression of acquired immune deficiency syndrome (AIDS)-like viral infection compared with Indian rhesus macaques (Trichel, Rajakumar \& Murphey-Corb, 2002). Prior to conducting studies utilising NHP models, it is therefore necessary to study their genetic background as NHP models originating from various geographical locations exhibit inter- and intraspecific genetic variations (Haus et al., 2014). Phylogenetic studies of the various populations of cynomolgus macaques across Indochina and Southeast Asia, have suggested the division of the cynomolgus macaques into two groups - namely the continental group consisting of Indochinese and Malaysian populations, and the insular group comprising of Indonesian, Bornean, and Philippine populations (Tosi \& Coke, 2007; Blancher et al., 2008; Rovie-Ryan et al., 2013). Based on mitogenomic data, further subdivisions were observed within the continental group that separates the Indochinese and Malaysian macaque populations (Liedigk et al., 2015). Owing to how the Peninsular Malaysian cynomolgus macaque have higher levels of nucleotide diversity and are monophyletic compared with other Southeast Asian cynomolgus macaque populations (Smith, McDonough \& George, 2007; Abdul-Latiff et al., 2014), we believe additional transcriptomic information of the wild Malaysian cynomolgus macaque will augment the existing cynomolgus macaque transcriptomic dataset for future biomedical researches, for instance, studies related to AIDS which rely on panels of immune function-related genes that are potentially expressed in lymph node, spleen, and/or thymus tissues.

.

(3)
harvested from cynomolgus macaques exposed to urban environments using the Illumina HiSeq 2500 platform. A reference-guided assembly was performed to assemble the sequencing reads by mapping the reads to the $M$. fascicularis reference genome, followed by empirical analysis of differential gene expression (EDGE test), and functional annotations and categorisation of the assembled reads. The dataset presented here will prove valuable for future immunological or drug translational studies involving cynomolgus macaques as NHPs.

\section{Materials and Methods}

\section{Ethics Statement}

The sampling and euthanasia of conflict $M$. fascicularis macaques were carried out by the Department of Wildlife and National Parks (DWNP) Malaysia (Permit No: JPHL\&TN(IP): 80- 
150

151

152

153

154

155

156

157

158

159

160

161

162

163

164

165

166

167

168

169

170

171

172

173

174

175

176

177

178

179

180

181

182

183

184

185

\section{0}

4/2) and were performed according to the guidelines set by the Institutional Animal Care and Use Committee (IACUC), University of California, Davis, United States of America as adopted by the PREDICT Project in Malaysia. Permission to obtain macaque lymph node, spleen, and thymus tissue samples for research purposes by the Genetics Lab at the Department of Biology was granted by DWNP.

Macaca fascicularis Sampling

Three male conflict $M$. fascicularis individuals from the state of Selangor in Malaysia were captured by DWNP. The three individuals were adolescents with total length of $79.0 \mathrm{~cm}$, $80.5 \mathrm{~cm}$, and $91.0 \mathrm{~cm}$ and weighing at $1.63 \mathrm{~kg}, 1.43 \mathrm{~kg}$, and $2.11 \mathrm{~kg}$ respectively, and appeared to belong in the same family group. Based on visual examination, the macaques were free of disease. Lymph node, spleen, and thymus tissues of the three individual macaques were harvested and stored separately in $1.5 \mathrm{ml}$ tubes filled with RNAlater RNA Stabilization Agent (Qiagen), which were incubated in $4^{\circ} \mathrm{C}$ overnight, and subsequently stored in $-80^{\circ} \mathrm{C}$.

\section{RNA Extraction \& Quality Check}

(1)

Total RNA samples were extracted from the nine harvested tissues using Qiagen's RNeasy Mini Kit. Due to the presence of genomic DNA in the RNA extract, a modified protocol was employed by utilizing Epicentre's DNase I solution to remove genomic DNA contamination. The intactness of the RNA extracts were visualised using 1\% agarose gel, and their respective concentrations quantitated using a Qubit fluorometer (Life Technologies, United States of America). The integrity of the RNA extracts were determined using Agilent's 2100 Bioanalyzer (Agilent Technologies, Inc., United States of America) via an RNA Pico chip. RNA samples with RIN of more than 7.0 were selected for the subsequent library preparation step.

RNA-Seq Library Preparation \& Sequencing

A total of nine sequencing libraries were prepared, with three replicates for each tissue type. The library preparation step consisted of two main sections; the removal of ribosomal RNA (rRNA) from the total RNA, and the conversion of the rRNA-depleted RNA to cDNA. rRNA depletion was carried out using the Ribo-Zero ${ }^{\mathrm{TM}}$ Gold Kit (Human/Mouse/Rat) (Epicentre, United States of America), while the conversion of treated RNA to cDNA was performed using 
186

187

188

189

190

191

192

193

194

195

196

197

198

199

200

201

202

203

204

205

206

207

208

209

210

211

212

213

214

215

216

217

218

219

220

221

222

223

the ScriptSeq ${ }^{\mathrm{TM}}$ v2 RNA-Seq Library Preparation Kit (Epicentre, United States of America). The size fragmentation distribution of the constructed cDNA library was assessed using Agilent's 2100 Bioanalyzer (Agilent Technologies, United States of America) via a High-Sensitivity DNA assay. The cDNA library was also subjected to quantitation via a qPCR assay using a CFX96 Touch $^{\text {TM }}$ Real-Time PCR Detection System (Bio-Rad, United States of America).The final normalised cDNA library was pooled together in a single reaction and was sequenced on Illumina's HiSeq 2500 platform in rapid run mode for $75 \times 2$ bp paired-end reads at the High Impact Research Centre in Universiti Malaya, Malaysia. Paired end sequencing data for lymph node, spleen, and thymus were submitted to NCBI Short Read Archive (SRA) database under accession SRP096937.

\section{Data Analysis}

Base quality and sequencing adapter trimming, reads mapping, biological replicates correlation analyses, and subsequent differential gene expression analyses were carried out on CLC Genomics Workbench v.7.5.1 (Qiagen, Denmark). For base quality checking and sequence adapter trimming, quality limit $=0.01$, or the equivalent of $\mathrm{Q}_{20}$, was utilised to remove bases with a quality value of less than 20 as well as sequencing adapters. The filtered reads were then mapped to the M. fascicularis reference genome (accession number: GCF_000364345.1) via reference guided assembly. Reads were set to map to "Gene Regions Only", including intron and exon regions of the genome. Expression levels were calculated as total number of reads mapped to a gene (Total Gene Reads).

A suite of biological replicates correlation analyses was performed, including normalisation, hierarchical clustering of samples, principal component analysis, and box plot analysis. Normalisation by total reads per million was performed using CLC Genomics Workbench's normalisation suite, and is similar to the counts per million (CPM) / transcripts per million (TPM) method of normalising RNA-Seq count data. Subsequent analyses including hierarchical clustering of samples, principal component analysis, and box plot analysis were performed using normalised expression values to determine the consistency of the gene expression profiles across replicates and tissue samples. For hierarchical clustering of samples, 1-Pearson correlation distance measure was employed together with average cluster linkage. Scatter plots of the gene expression profiles between biological replicates were generated for the principal component analysis based on normalised expression values (Figures S1-S6). Pearson correlation values (r-value) were generated from the scatter plot.

\section{Differential Gene Expression Analysis}


225

226

227

228

229

230

231

232

233

234

235

236

237

238

239

240

241

242

243

244

245

246

247

248

249

250

251

252

253

254

255

256

257

258

259

Empirical analysis of differentially expressed genes (EDGE test) was carried out on normalised expression profiles to identify differentially expressed genes between the lymph node, spleen, and thymus tissue samples. Three separate tissue comparisons were performed spleen vs. lymph, thymus vs. lymph, and spleen vs. thymus. Differentially expressed genes were filtered by selecting for genes with normalised fold change values of $>2$ or $<-2$. Selection of significantly differentially expressed genes were performed by filtering for genes with FDR corrected p-value $<0.05$.

Gene Ontology and KEGG Pathway Annotation \& Categorisation

Genes were assigned Gene Ontology and KEGG pathway annotations by applying gene ontology and pathway association files (.gaf2) obtained from the UniProtKB and KEGG databases respectively. Functional and pathway categorisation of expressed genes (normalised expression value $>1$ ) were carried out using a suite of online databases. GO categorization was carried out via Panther Database version 11.1 released on 2016-10-24 (http://pantherdb.org/) utilising Macaca mulatta as the reference organism. Classifications of genes into biological process, molecular function, and cellular component GO categories were visualised via the "Functional classification viewed in pie chart" analysis. For KEGG pathway categorisations, DAVID Bioinformatics Resources version 6.8 (https://david-d.ncifcrf.gov/home.jsp) was utilised with the M. fascicularis as the background organism. EASE threshold score was set at 0.01 when determining significantly enriched pathway categories. KEGG pathway mappings with highlighted mapped objects were retrieved from KEGG mapper version 2.8 tool in the KEGG website (http://www.kegg.jp/kegg/mapper.html).

Tissue-specific Genes

To identify and visualise tissue-specific genes in lymph node, spleen, and thymus tissues, expressed genes for each tissue type were input to the web tool Venn Diagrams (http://bioinformatics.psb.ugent.be/webtools/Venn).

RNA-Seq Data Validation

(1)

Validation of RNA-Seq gene expression profiles were performed using NanoStringnCounter XT gene expression assay (NanoString Technologies Inc., Seattle, WA, USA). A total of 24 genes were selected for validation based on fold change patterns and 
260

261

262

263

264

265

266

267

268

269

270

271

272

273

274

275

276

277

278

279

280

281

282

283

284

285

286

287

288

289

290

291

292

293

294

295

296

297

immune-related functions. An additional three housekeeping genes (ACTB, GAPDH, and $Y W H A Z$ ) were also selected for reference genes normalising of digital counts data. Fold change patterns for 24 genes in their respective tissue comparisons were compared between the RNASeq and NanoString platforms. Custom CodeSet probes were designed from M. fascicularis FASTA sequences obtained from the reads mapping assembly from this study. Table S1 lists the genes utilised for the RNA-Seq data validation and their respective probe pair sequences. The amount of total RNA utilised for the hybridisation step was $200 \mathrm{ng}$. Digital scanning of RNAprobe hybrids were performed at High 280 Field of View (FOV) setting. Hybridisation and digital scanning of the RNA-probe hybrids were performed at High Impact Research Centre in Universiti Malaya, Malaysia.

\title{
Results
}

\section{Sequence Reads Filtering \& Mapping}

\begin{abstract}
We investigated the transcriptome of three tissues in triplicates obtained from the Malaysian M. fascicularis using the RNA-Seq platform. The number of raw sequence reads obtained from the high-throughput sequencing of lymph node, spleen, and thymus tissues were $50,180,478,63,956,392$, and 60,071,208 respectively with a sequence length of 70 base pairs for each read. Raw sequence reads were trimmed and filtered to remove PhiX Illumina positive control sequences, adapter and index barcode sequences, and low quality sequences based on base quality $\mathrm{Q}_{20}$. Post-trimming, the number of sequence reads for lymph node, spleen, and thymus tissues were 47,559,293, 60,285,505, and 56,583,352 respectively, with the average length of the sequence reads ranging from 57.5 base pairs to 60.3 base pairs. The percentage of reads that were retained post-trimming ranged from $96.52 \%$ to $97.73 \%$, indicating that at least $96 \%$ of the reads were above base quality $Q_{20}$ and were suitable for reference-guided assembly.
\end{abstract}

The filtered reads from each replicate were then mapped to 36,233 genes within the $M$. fascicularis reference genome obtained from NCBI (Accession: GCF_000364345.1). The total number of paired and broken paired reads mapped to the reference genome for lymph, spleen, and thymus samples are 25,476,162, 37,536,104, and 30,782,715 reads respectively. For the counting of fragments mapped to exon and intron regions, only paired end reads were taken into account. The overall mapping percentage (sum of percentage of reads mapped in pairs and broken pairs) ranged from $53.00 \%$ to $63.00 \%$ with lymph showing lower overall mapping percentage compared to spleen and thymus. Table 1 summarises the filtering and mapping statistics of lymph node, spleen, and thymus sequence reads. Supplementary information on the individual mapping statistics for each individual library are included in Table S2. 
307

Table 1: Filtering and mapping statistics of lymph, spleen, and thymus sequence reads to Macaca fascicularis reference genome (GCF_000364345.1). Reads were mapped to gene regions only with a maximum number of hits for a read $=10$. Only intact paired reads were taken into account when counting the fragments by type.

\begin{tabular}{|l|c|c|c|}
\hline \multirow{2}{*}{} & \multicolumn{3}{|c|}{ Tissue } \\
\cline { 2 - 4 } & Lymph & Spleen & Thymus \\
\hline Reads (Before trim) & $50,180,478$ & $63,956,392$ & $60,071,208$ \\
\hline Reads (After trim) & $47,559,293$ & $60,285,505$ & $56,583,352$ \\
\hline $\begin{array}{l}\text { Reads uniquely mapped to exon } \\
\text { regions }\end{array}$ & $9,712,306$ & $14,061,540$ & $9,520,236$ \\
\hline $\begin{array}{l}\text { Reads uniquely mapped to } \\
\text { intron regions }\end{array}$ & $7,508,538$ & $10,242,700$ & $6,674,232$ \\
\hline Reads mapped in pairs (\%) & 38.44 & 42.66 & 31.47 \\
\hline $\begin{array}{l}\text { Reads mapped in broken pairs } \\
\text { (\%) }\end{array}$ & 15.13 & 19.60 & 22.93 \\
\hline Overall mapping percentage (\%) & 53.57 & 62.26 & 54.40 \\
\hline
\end{tabular}

\title{
Biological Replicates Correlation Analysis
}

\begin{abstract}
Biological replicates correlation analysis of lymph node, spleen, and thymus tissues show consistent expression values in the three replicates for each tissue type (Pearson correlation value, $r>0.8$ ). No outliers were detected and excluded from the subsequent analyses. Principal component analysis scatter plots and box plots of the normalised expression values were also generated and included in the Supplementary Data (Figures S1-S6).
\end{abstract}

\section{Differential Gene Expression Analysis}

(1)

Three tissue comparison experiments were set up, namely Spleen vs. Lymph, Thymus vs. Lymph, and Spleen vs. Thymus. The total of differentially expressed genes called for Spleen vs. Lymph, Thymus vs. Lymph, and Spleen vs. Thymus were 574, 5,402, and 7,008 genes respectively. In Spleen vs. Lymph comparison, CCL17 and AIF1L were among the top regulated genes in lymph and spleen respectively. For the Thymus vs. Lymph tissue comparison, the genes CCR7 and PRH2 were the top regulated genes in lymph and thymus respectively. Lastly, for Spleen vs. Thymus comparison, MYO7A and KLK1 were among the top highest regulated genes 
323 for spleen and thymus respectively. Table S3 lists the differentially expressed genes with their 324 annotated gene names and descriptions.

325

326

327

328

329

330

331

332

333

335

336

337

338

339

340

341

342

343

344

345

346

347

348

349

350

351

352

To assign Gene Ontology (GO) and KEGG pathway annotations to the genes, gene ontology and pathway annotation files (.gaf2) were obtained from UniProtKB and KEGG database respectively and imported to CLC Genomics Workbench. The numbers of genes with gene ontology and pathway annotations are listed in Table 2.

Table 2: Total number of genes with Gene Ontology (Biological Process, Molecular Function, and Cellular Component) and KEGG pathway annotations.

\begin{tabular}{|c|c|c|c|c|}
\hline \multirow{2}{*}{ Annotation Category } & \multicolumn{3}{|c|}{ Gene Ontology } & \multirow{2}{*}{ KEGG } \\
\cline { 2 - 4 } & $\begin{array}{c}\text { Biological } \\
\text { Process }\end{array}$ & $\begin{array}{c}\text { Molecular } \\
\text { Function }\end{array}$ & $\begin{array}{c}\text { Cellular } \\
\text { Component }\end{array}$ & Pathway \\
\hline $\begin{array}{c}\text { Number of } \\
\text { Annotated Genes }\end{array}$ & 12,626 & 12,637 & 13,230 & 5387 \\
\hline
\end{tabular}

To classify the genes into GO categories, expressed genes were input to the Panther Database. For lymph node tissue, 15,611, 7876, and 6141 genes were categorised to more than one GO categories in the Biological Process, Molecular Function, and Cellular Component domains respectively. In the spleen tissue, 14,838, 7465, and 5892 genes were categorised to more than one GO categories in the Biological Process, Molecular Function, and Cellular Component domains respectively. While in the thymus tissue, 12,161, 6143, and 5029 genes were categorised to more than one GO categories in the Biological Process, Molecular Function, and Cellular Component domains respectively. The distribution of the genes into different level 2 GO categories are shown in Table 3. The Biological Process category with the highest number of expressed genes for all three tissues is Cellular Process (Lymph, 4429; Spleen, 4239; Thymus, 3551). For Molecular Function GO domain, the Catalytic Activity category contains the highest number of expressed genes for all three tissues (Lymph, 2972; Spleen, 2856; Thymus, 2411). As for Cellular Component, all three lymph, spleen, and thymus tissues show the highest number of expressed genes in the Cell Part category (Lymph, 2339; Spleen, 2247; Thymus, 2002).

Table 3: Categorisation of expressed genes to Gene Ontology terms in lymph node, spleen, and thymus tissues.

Gene Ontology

NUMBER OF GENES

\begin{tabular}{l|l|l|} 
LYMPH & SPLEEN & THYMUS \\
\hline
\end{tabular}




\begin{tabular}{|c|c|c|c|}
\hline & NODE & & \\
\hline \multicolumn{4}{|l|}{ BIOLOGICAL PROCESS } \\
\hline Cellular Process & 4429 & 4239 & 3551 \\
\hline Metabolic Process & 3793 & 3634 & 3155 \\
\hline Localization & 1152 & 1101 & 931 \\
\hline Response to Stimulus & 1216 & 1150 & 856 \\
\hline Developmental Process & 1067 & 983 & 786 \\
\hline Biological Regulation & 1064 & 1006 & 758 \\
\hline Cellular Component Organization or Biogenesis & 907 & 875 & 772 \\
\hline Multicellular Organismal Process & 790 & 732 & 526 \\
\hline Immune System Process & 611 & 573 & 414 \\
\hline Biological Adhesion & 284 & 270 & 209 \\
\hline Reproduction & 186 & 166 & 118 \\
\hline Locomotion & 92 & 92 & 73 \\
\hline Rhythmic Process & 10 & 8 & 5 \\
\hline Growth & 4 & 4 & 4 \\
\hline Cell Killing & 6 & 5 & 3 \\
\hline \multicolumn{4}{|l|}{ MOLECULAR FUNCTION } \\
\hline Catalytic Activity & 2972 & 2856 & 2411 \\
\hline Binding & 2957 & 2806 & 2369 \\
\hline Transporter Activity & 630 & 589 & 447 \\
\hline Receptor Activity & 561 & 518 & 338 \\
\hline Structural Molecule Activity & 502 & 463 & 407 \\
\hline Signal Transducer Activity & 186 & 170 & 115 \\
\hline Translation Regulator Activity & 32 & 32 & 31 \\
\hline Antioxidant Activity & 19 & 15 & 14 \\
\hline Channel Regulator Activity & 17 & 16 & 11 \\
\hline \multicolumn{4}{|l|}{ CELLULAR COMPONENT } \\
\hline Cell Part & 2339 & 2247 & 2002 \\
\hline Organelle & 1458 & 1397 & 1247 \\
\hline Membrane & 957 & 914 & 697 \\
\hline Macromolecular Complex & 774 & 761 & 686 \\
\hline Extracellular Region & 392 & 365 & 247 \\
\hline Extracellular Matrix & 125 & 113 & 79 \\
\hline Cell Junction & 61 & 59 & 43 \\
\hline Synapse & 35 & 36 & 28 \\
\hline
\end{tabular}

Expressed genes were also input to DAVID Bioinformatics Resources to classify the genes into KEGG pathway categories (Table 4). In the lymph node, spleen, and thymus, 4848, 4658 , and 3893 genes respectively were assigned to one or more KEGG pathway categories. For lymph node, spleen, and thymus, the Global overview and maps pathway umbrella category contains the highest number of expressed genes, followed by the Signal transduction category. 
360 Table 4: KEGG pathway term distribution of expressed genes in lymph node, spleen, and thymus 361 tissues.

\begin{tabular}{|c|c|c|c|}
\hline \multirow[b]{2}{*}{ KEGG Pathway Terms } & \multicolumn{3}{|c|}{ NUMBER OF GENES } \\
\hline & $\begin{array}{l}\text { LYMPH } \\
\text { NODE }\end{array}$ & SPLEEN & THYMUS \\
\hline \multicolumn{4}{|l|}{ METABOLISM } \\
\hline Global overview and maps & 947 & 903 & 776 \\
\hline mcf01100:Metabolic pathways & 947 & 903 & 776 \\
\hline Carbohydrate metabolism & $\mathbf{0}$ & $\mathbf{0}$ & 89 \\
\hline mcf00562:Inositol phosphate metabolism & 0 & 0 & 52 \\
\hline mcf00620:Pyruvate metabolism & 0 & 0 & 37 \\
\hline Energy metabolism & 145 & 141 & 136 \\
\hline mcf00190:Oxidative phosphorylation & 145 & 141 & 136 \\
\hline Glycan biosynthesis and metabolism & $\mathbf{0}$ & $\mathbf{0}$ & 36 \\
\hline mcf00510:N-Glycan biosynthesis & 0 & 0 & 36 \\
\hline \multicolumn{4}{|l|}{ GENETIC INFORMATION PROCESSING } \\
\hline Folding, sorting and degradation & $\mathbf{0}$ & $\mathbf{0}$ & 185 \\
\hline mcf03018:RNA degradation & 0 & 0 & 61 \\
\hline $\begin{array}{l}\text { mcf04141:Protein processing in endoplasmic } \\
\text { reticulum }\end{array}$ & 0 & 0 & 124 \\
\hline \multicolumn{4}{|l|}{$\begin{array}{l}\text { ENVIRONMENTAL INFORMATION } \\
\text { PROCESSING }\end{array}$} \\
\hline Membrane transport & 39 & 39 & $\mathbf{0}$ \\
\hline mcf02010:ABC transporters & 39 & 39 & 0 \\
\hline Signal transduction & 531 & 439 & 605 \\
\hline mcf04010:MAPK signalling pathway & 194 & 191 & 164 \\
\hline mcf04012:ErbB signalling pathway & 71 & 70 & 67 \\
\hline mcf04014:Ras signalling pathway & 0 & 0 & 142 \\
\hline mcf04015:Rap1 signalling pathway & 0 & 0 & 140 \\
\hline mcf04064:NF-kappa B signalling pathway & 72 & 72 & 0 \\
\hline mcf04310:Wnt signalling pathway & 110 & 106 & 92 \\
\hline mcf04668:TNF signalling pathway & 84 & 0 & 0 \\
\hline Signalling molecules and interaction & 77 & 74 & $\mathbf{0}$ \\
\hline
\end{tabular}




\begin{tabular}{|c|c|c|c|}
\hline mcf04512:ECM-receptor interaction & 77 & 74 & 0 \\
\hline \multicolumn{4}{|l|}{ ORGANISMAL SYSTEM } \\
\hline Development & 102 & 100 & 93 \\
\hline mcf04360:Axon guidance & 102 & 100 & 93 \\
\hline Immune system & 266 & 221 & 138 \\
\hline mcf04611:Platelet activation & 107 & 104 & 87 \\
\hline mcf04612:Antigen processing and presentation & 61 & 60 & 0 \\
\hline mcf04662:B cell receptor signalling pathway & 57 & 57 & 51 \\
\hline $\begin{array}{l}\text { mcf04672:Intestinal immune network for IgA } \\
\text { production }\end{array}$ & 41 & 0 & 0 \\
\hline Nervous system & 86 & 178 & 90 \\
\hline mcf04722:Neurotrophin signalling pathway & 0 & 95 & 90 \\
\hline mcf04725:Cholinergic synapse & 86 & 83 & 0 \\
\hline Endocrine system & 208 & 203 & 303 \\
\hline mcf04910:Insulin signalling pathway & 0 & 0 & 99 \\
\hline mcf04915:Estrogen signalling pathway & 83 & 81 & 74 \\
\hline mcf04917:Prolactin signalling pathway & 0 & 0 & 50 \\
\hline mcf04921:Oxytocin signalling pathway & 125 & 122 & 0 \\
\hline mcf04919:Thyroid hormone signalling pathway & 0 & 0 & 80 \\
\hline \multicolumn{4}{|l|}{ CELLULAR PROCESSES } \\
\hline Transport and catabolism & $\mathbf{0}$ & 68 & 325 \\
\hline mcf04142:Lysosome & 0 & 0 & 90 \\
\hline mcf04144:Endocytosis & 0 & 0 & 172 \\
\hline mcf04146:Peroxisome & 0 & 68 & 63 \\
\hline Cellular community - eukaryotes & 174 & 172 & 151 \\
\hline mcf04510:Focal adhesion & 174 & 172 & 151 \\
\hline
\end{tabular}

The total number of expressed genes enriched in the KEGG pathway Immune system category for lymph node, spleen, and thymus were 266, 221, and 138 genes respectively. The four Immune system pathways that were enriched include Platelet activation pathway, Antigen processing and presentation, B cell receptor signalling pathway, and Intestinal immune network for IgA production. Genes enriched in both Platelet activation and B cell receptor signalling pathways are expressed in lymph node, spleen, and thymus tissues. For the Platelet activation pathway, 87 genes were expressed in all three tissues, including TLN1, TLN2, FERMT3, ITGB3, $F G A, F G B$, and $F G G$ which are involved in the activation and aggregation of platelets. Genes involved in the $\mathrm{B}$ cell receptor signalling pathway had 51 genes expressed across all tissues, 
372

373

374

375

376

377

378

379

380

381

382

383

384

385

386

387

388

389

390

391

392

393

394

395

396

397

398

399

400

401

402

403

404

405

406

407

408

409

410

which include genes such as $S Y K, P I K 3 A P 1, A K T 1, A K T 2, A K T 3, C H U K, I K B K B$, and IKBKG that also take part in the PI3K-Akt signalling pathway and NF-kappa B signalling pathway. Genes enriched in the Antigen processing and presentation pathway were expressed in both lymph node and spleen tissues with 61 and 60 genes respectively. All 60 lymph genes expressed in the Antigen processing and presentation pathway were co-expressed in spleen, with genes involved in the MHC I (PSME1, PSME2, PSME3, TAP1, TAP2) and MHC II (CD74, LGMN, $C T S S$ ) pathways. The Intestinal immune network for IgA production pathway was specifically enriched in lymph node, with 41 genes enriched in this pathway including genes such as IL5, IL6, IL10,TGFB1, TNFSF13, and AICDA which are involved in the proliferation of B cells into $\operatorname{IgA}+\mathrm{B}$ cells.

Thymus has the highest number of pathways that are specifically enriched with genes. The enriched pathways include Inositol phosphate metabolism, Pyruvate metabolism, N-Glycan biosynthesis, RNA degradation, Protein processing in endoplasmic reticulum, Ras signalling pathway, Rap1 signalling pathway, Insulin signalling pathway, Prolactin signalling pathway, Thyroid hormone signalling pathway, Lysosome, and Endocytosis. A group of related pathways were observed to be involved in the regulation of the immune system, which include N-Glycan biosynthesis, Protein processing in endoplasmic reticulum, Ras signalling pathway, Rap1 signalling pathway, and RNA degradation pathway). The TNF signalling pathway was the only tissue-specifically enriched pathway in lymph node, with a total of 84 genes enriched in the pathway. Enriched genes such as TNFRSF1A, TNFRSF1B, MAP3K7, TAB1, JUN, TRAF1, $T R A F 3, B I R C 2$ and $B I R C 3$ directly participate in the activation of leukocytes, inflammatory cytokines, and cell survival. All KEGG pathway figures are located in Supplementary Data, Figures S7-S15.

\section{Genes Involved in Immune-related Pathways}

The Platelet activation pathway is activated by the adhesion of ADP to purinergic receptor $\mathrm{P} 2 \mathrm{Y}$ that begins the complement and coagulation cascade involving genes TLN2, FERMT3, ITGB3, FGA, FGB, and FGG at the end of the cascade. TLN2 codes for the cytoskeleton protein Talin-2 and is a component that links actin cytoskeleton with integrin and catalyses focal adhesion signalling pathways (Zhang et al., 2008). FERMT3, together with TLN1 and TLN2, activates integrins beta-3 (ITGB3), and when activated, triggers platelet-platelet interactions via binding of fibrinogens alpha, beta, and gamma. These fibrinogens (respectively encoded by $F G A, F G B$, and $F G G$ ) interact to form a polymerisation that form a fibrin matrix that physically plugs ruptured endothelial surface.

In the $\mathrm{B}$ cell receptor signalling pathway, a response to foreign antigen binding to the $\mathrm{B}$ cell receptor (BCR) is for the gene $S Y K$ to encode enzyme tyrosine kinase. In effect, tyrosine 
411

412

413

414

415

416

417

418

419

420

421

422

423

424

425

426

427

428

429

430

431

432

433

434

435

436

437

438

439

440

441

442

443

444

445

446

447

448

449

kinase activates the phosphatidylinositol 3'-kinase (PI3K)-Akt signalling pathway via the BCAP signalling adapter encoded by PIK3AP1. The PI3K-Akt signalling pathway then activates the AKT kinase coded by $A K T 1, A K T 2$, and $A K T 3$ that functions to ensure cell survival by reducing oxidative stress acting on the cell, thus preventing cell apoptosis. AKT kinase also activates the Nuclear factor-kappa B (NF-kappa B) signalling pathway via the IKK enzyme complex (coded by $C H U K, I K B K B$, and $I K B K G$ ) which activate genes that further ensures cell survival (Faissner et al., 2006).

In the MHC I pathway, genes PSME1, PSME2, and PSME3 encode for proteasome activator PA28, an immunoproteasome involved in the Antigen processing and presentation pathway. Genes TAP1 and TAP2 encode for antigen transporters 1 and 2 respectively, and is located on the membrane of the endoplasmic reticulum of a cell. PA28 activator complex play a role in presenting antigens to the antigen transporters, which antigen transport the antigens from cytoplasm to the endoplasmic reticulum to be presented to the MHC I molecules. The CD74 gene is significant in the MHC II pathway for its antigen processing regulatory function. It codes for HLA class II histocompatibility antigen gamma chain protein that binds to the invariant chain (Ii) polypeptide to form the MHC II protein complex. The complex is then migrated to the endoplasmic reticulum of the cell where the complex is cleaved by legumain (coded by $L G M N$ ) and cathepsin S (coded by CTSS) to a smaller and more stable form of MHC II called CLIP that is ultimately presented on the cell surface.

In the Intestinal immune network for IgA production pathway, as MHC II proteins recognise and present antigens to the A proliferation-inducing ligand (APRIL) receptor (coded by TNFSF 13), a cascade begins that ultimately differentiate $\mathrm{B}$ cells into immunoglobulin $\mathrm{A}+\mathrm{B}$ cells. Cytokines interleukin-5, interleukin-6, and interleukin-10 (coded by IL5, IL6, and IL10 respectively) together with transforming growth factor beta-1 (coded by TGFB1) regulate the proliferation of $\mathrm{B}$ cells and eosinophils. The $A I C D A$ gene that codes for activation induced cytidine deaminase enzyme, also facilitates the class-switch of B cells into IgA+ B cells.

\section{Lymph Node-specific Pathway}

Two pathways are enriched in the TNF signalling pathway involving genes TNFRSF1A and TNFRSF1B that code for the TNFR1 and TNFR2 receptors respectively. Each receptor, when bound with their respective ligands activate two separate pathways with different functions. When the TNFR1 receptor is activated, a signalling cascade activates the MAPK signalling pathway cascade which is mediated by the expression of the TAK1-TAB1 kinase complex coded by genes $M A P 3 K 7$ and $T A B 1$. The activation of the MAPK signalling pathway ultimately regulates the expression of Transcription factor AP-1 (coded by $J U N$ ), which is known to play a role in apoptosis, cytokine regulation, and leukocyte activation (Foletta, Segal \& 
450

451

452

453

454

455

456

457

458

459

460

461

462

463

464

465

466

467

468

469

470

471

472

473

474

475

476

477

478

479

480

481

482

483

484

485

486

487

488

489

\section{3}

Cohen, 1998; Kappelmann, Bosserhoff \& Kuphal, 2014). The activation of the TNFR2 receptor by the ligand LTA activates a signalling cascade that is mediated by TNF receptor associated factors 1 and 3 (coded respectively by TRAF1 and TRAF3). The TNF receptor associated factors then recruit Apoptosis inhibitors 2 and 3 (coded by BIRC2 and BIRC3) to ensure cell survival (Wang et al., 2012).

Thymus-specific Immune-related Pathways

\section{Thymus-specific Immune-related Pathways}

Out of the three tissues, thymus has the highest number of pathways that are specifically enriched with genes. A total of 984 genes were distributed across 12 pathway categories as analysed with DAVID Bioinformatics Resources using the Macaca fascicularis genome reference as the background. Five immune-related pathways were identified, which include Ras signalling pathway, Rap1 signalling pathway, RNA degradation, N-Glycan biosynthesis pathway, and Protein processing in endoplasmic reticulum. Two enriched pathway categories, the Ras signalling pathway and Rap1 signalling pathway, each had 142 and 140 genes expressed in their pathways. The Ras signalling pathway functions as a binary molecular switch to regulate cellular processes such as cell proliferation, migration, differentiation, or growth. Several other pathways are activated by the Ras pathway, and based on the enrichment analysis of the expressed macaque thymus genes, the Mitogen-activated protein kinase (MAPK) cascade, and the Rap1 signalling pathway were both activated by the Ras signalling pathway. The Rap1 signalling pathway also acts as a molecular switch to activate cellular processes including the MAPK cascade. These linked pathways suggest a role in the proliferation and differentiation of $\mathrm{T}$ cells in the thymus, as the $\mathrm{T}$ cell receptor signalling pathway precedes both the Ras and Rap1 signalling pathways. The expressed genes as illustrated in Figures S12 and S13 suggest that the T cells were undergoing proliferation and differentiation in the individual macaques, however the lack of expressed genes enriched in the $\mathrm{T}$ cell receptor signalling pathway and also the lack of the activation of $\alpha \beta \mathrm{T}$ cell receptor (TCR) in both Ras and Rap1 signalling pathways further suggests that the proliferation and differentiation of the $\mathrm{T}$ cells was not caused by interactions with foreign antigens. The Ras signalling pathway is also known to activate apoptosis functions in a cell, and based on the expression of genes RASSF1, RASSF5, and STK4 in the Ras signalling pathway, it is suggested that the apoptosis function is switched on and likely functions in the cell death of thymocytes that fail the positive and negative selections during the developmental process.

ex

The enrichment of RNA degradation pathway suggests the upregulation of eukaryotic exosomes in the macaque transcriptome. Exosomes have multiple roles in the cellular environment, including cell-cell communication, cellular waste removal, quality control of nuclear RNA, and antiviral defence (Schmid \& Jensen, 2008). Exosomes have been shown to be released by dendritic, $\mathrm{B}$, and $\mathrm{T}$ lymphocytes during adaptive and innate immune responses (Alenquer \& Amorim, 2015). The multiprotein eukaryotic core exosome complex is coded by 
490

491

492

493

494

495

496

497

498

499

500

501

502

503

504

505

506

507

508

509

510

511

512

513

514

515

516

517

518

519

520

521

522

523

genes EXOSC1, EXOSC2, EXOSC3, EXOSC4, EXOSC4, EXOSC6, EXOSC7, EXOSC8, and EXOSC 9. Co-factors associated with the RNA exosome that are involved in RNA degradation include the Nuclear exosome co-factor, RRRP44 (coded by DIS3) and the Cytoplasmic exosome co-factor DIS3 Like Exosome 3'-5' Exoribonuclease (coded by DIS3L). These co-factors play a role in degrading specific target RNAs, for instance, DIS3L activates the RNA exosome complex to degrade unstable mRNAs in the cytoplasm (Staals et al., 2010), while DIS3 functions as surveillance for aberrant RNAs (Mollestonet al., 2016). The TRAMP complex consists of proteins poly(A) polymerase (coded by $P A P D 5, P A P D 7$ ), zinc-knuckle putative RNA-binding protein (coded by ZCCHC7), and RNA helicase (coded by SKIV2L2), and is an exosome coactivator complex that interacts with exosomes in the nucleus of eukaryotic cells to initiate 3' end processing and degradation of ribosomal RNA and small nucleolar RNA (Jia et al., 2011).

Other immune-related pathways include the N-Glycan biosynthesis pathway that controls the differentiation of immune-related glycoproteins. Proper folding of glycoproteins such as antigen receptors ( $\mathrm{T}$ and $\mathrm{B}$ cell receptors) and $\mathrm{MHC}$ molecules occur in the endoplasmic reticulum and correctly folded proteins are out of the ER via golgi body. The folding and transport of proteins involved it the differentiation of immune cells occur in the Protein processing in endoplasmic reticulum pathway. Folding of protein products are promoted by the protein Calreticulin, encoded by the gene CALR (Nauseef, McCormick \& Clark, 1995), while the selection and transport of proteins out of the ER is encoded by genes such as $L M A N 1, P R E B$, and SAR1A (Weissman, Plutner \& Balch, 2001; Nufer et al., 2003; Watson et al., 2006).

\section{Tissue-Specific Genes}

Venn diagram comparisons for all expressed genes illustrates the overlap of expressed genes among the lymph node, spleen, and thymus tissues (Figure 1). In the lymph node tissue, a total of 2630 genes were lymph node-specific genes, with CLEC4G, CCL20, SHOX2, SHISA3, and LOC102127387 being the top five most expressed lymph node-specific genes. For the spleen tissue, 253 genes were spleen-specific genes, with PPBP, NKX2-3,TCF21, CAMK2N1, and LOC102119108 being the top five most expressed spleen-specific genes. Thymus tissue has 279 thymus-specific genes with PRB3,KLK1, ZG16B, HTN1, LOC102142723 as the top five most abundantly expressed thymus-specific genes. A complete list of tissue-specific genes in their respective tissues are listed in Table S4. 


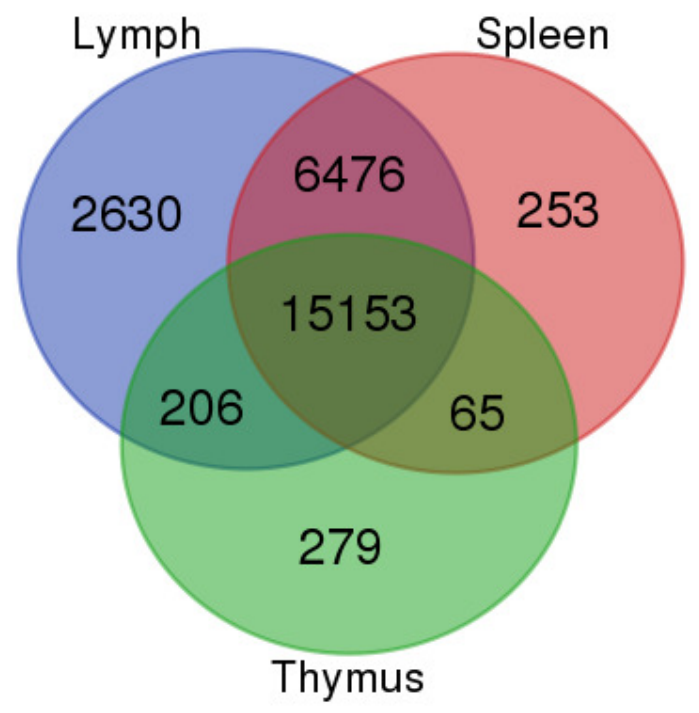

525

526 Figure 1: Venn diagram of the number of expressed genes (normalised expression values $>1$ ) in their respective tissues. A total of 15,153 genes were expressed in all three lymph node, spleen, and thymus tissues. The number of tissue-specific genes for lymph node, spleen, and thymus are 2630,253 , and 279 respectively.

Top Highly Expressed Tissue-Specific Genes for Each Tissue

532

533

534

535

536

537

538

539

540

541

542

543

544

545

546

547

548
In the lymph node tissue, CLEC4G, CCL20, SHOX2, SHISA3, and LOC102127387 are the top five most expressed lymph node-specific genes. Gene $C L E C 4 G$ is expressed in liver and lymph node sinusoidal endothelial cells, and codes for C-type lectin domain family 4 member $\mathrm{G}$ protein. Previous studies have identified the protein as a glycan-binding receptor, and is suggested to be involved in cell-cell adhesion processes and acts as a receptor for antigen clearance in lymph nodes (Liu et al., 2004). CCL20 codes for chemokine C-C motif ligand 20 that acts as a ligand for chemokine C-C receptor CCR6. The CCL20-CCR6 ligand-receptor pair is responsible for the chemotaxis of lymphocytes, neutrophils, proinflammatory IL17 producing helper T-cells (Th17), and the regulatory T-cells in response to inflammation (Frick et al., 2016). SHOX2, a gene which codes for Short stature homeobox protein 2, is a transcriptional factor involved in various embryological development processes (Branchi et al., 2016), and is implicated in Turner syndrome, whereby gene knockout of SHOX2 in mice results in skeletal growth abnormalities and short stature phenotypes (Gu et al., 2008). SHISA3, which codes for protein shisa-3 homolog, is a member of the Shisa family of proteins that modulate WNT and FGF signalling in developmental processes (Chen et al., 2014). 
For the spleen tissue, the top five most expressed spleen-specific genes are $P P B P, N K X 2-$ 3, TCF21, CAMK2N1, and LOC102119108. PPBP (also known as CXCL7) codes for Proplatelet basic protein, a platelet-derived growth-factor that acts as a chemoattractant and activator of neutrophils, and is involved in blood clotting and inflammation (Herring et al., 2015). Homeobox protein Nkx-2.3 is coded by $N K X 2-3$, while Transcription factor 21 is coded by TCF21. Both NKX2-3 and TCCF21 are transcription factors that are suggested to be significant in the organogenesis of the spleen (Brendolan et al., 2007; Czömpöly et al., 2011). CAMK2N1 codes for Calcium/calmodulin-dependent protein kinase II inhibitor 1 which inhibits Calcium/calmodulin-dependent protein kinase II (CaMKII) activity pertinent to cell growth processes (Wang et al., 2008).

The top five most expressed thymus-specific genes include PRB3, KLK1, ZG16B, HTN1, and LOC102142723. PRB3 gene codes for Proline Rich Protein BstNI Subfamily 3, which acts as a receptor for Gram-negative bacterium Fusobacterium nucleatum (Gillece-Castro et al., 1991). KLK1 codes for Kallikrein Serine Protease 1, and is suggested to have an antiinflammatory role in the adaptive immune system by promoting lymphocyte proliferation (Panos et al., 2014). The gene $Z G 16 B$ codes for Zymogen Granule Protein 16B, and functions to regulate chemokine CXCR4 and acts as an endogenous ligand of Toll-like receptor 2 (TLR2) and TLR4 for the expression of chemokines CCL5 and Macrophage migration inhibitory factor (MIF) (Park et al., 2011). HTN1 codes for protein Histatin-1, and is involved in antifungal and antibacterial activities (Oppenheim et al., 2007).

\section{Validation of RNA-Seq Differential Gene Expression}

Twenty four genes were selected to validate the differential gene expression data obtained from the RNA-Seq platform. Fold change values from RNA-Seq platform were compared with fold change values obtained from the NanoString nCounter XT platform. Figure 2 provides a graphical comparison of $\log _{2}$ transformed fold change values between the two platforms. The concordant fold change directions of all genes confirms the differential gene expression results from the RNA-Seq platform. The NanoString gene expression assay utilises barcoded probes that hybridise to their specific RNA target and are directly counted via the colour-coded probes. This eliminates the need to enzymatically amplify the template and also minimises pipetting procedures to reduce errors and contaminations. The platform reliably captures gene expression profiles effectively with relatively small amounts of template RNA (100ng) and also serve as an alternative to RT-qPCR gene expression assays (Radke et al., 2014; $\mathrm{Hu}$ et al., 2016). Previous cynomolgus macaque studies utilised RT-PCR and RT-qPCR to validate their high-throughput sequencing results, and as such, the present study is the first to validate cynomolgus macaque RNA-Seq data using the NanoString nCounter XT gene expression assay. The platform's relative ease of use, quick turnaround, reproducibility, and 
588 sensitivity make it suitable for medium to high-throughput screening of panels of genes for 589 future biomedical science research utilising the cynomolgus macaque as NHP models. 
AIF1L

CLEC4G

GPR182

LIFR

MEOX2

NKX2-3

SHISA3

TXK

ADRB1

CEBPD

CLEC2D

CTSS

FOXQ1

PIK3R5

PLCB1

PRH2

CCL28

CD5L

DVL1

FCGR2A

GBP1

KLK1

MARCKSL1

MYO7A
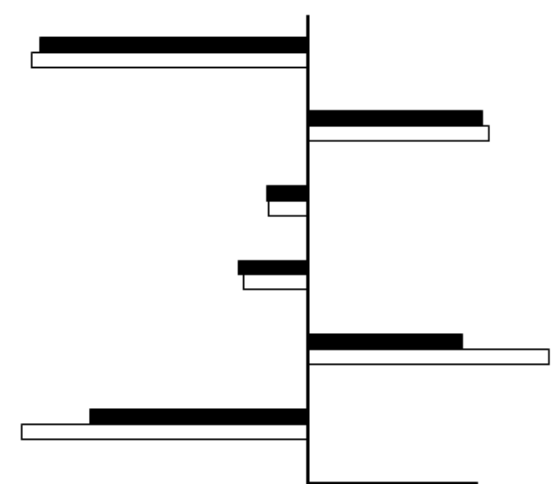

号

RNA-Seq $\square$ NanoString

592 Figure 2: Validation of RNA-Seq differential gene expression results. $\log _{2}$ transformed fold

593 change values from three sets of tissue comparisons (Spleen vs. Lymph; Thymus vs. Lymph; 
594 595

596

597

598

599

600

601

602

603

604

605

606

607

608

609

610

611

612

613

614

615

616

617

618

619

620

621

622

623

624

625

626

627

628

629

630

631

632

633

Spleen vs. Thymus) were obtained from RNA-Seq and NanoString nCounter XT platforms. Solid bar represents fold change values obtained from RNA-Seq platform, while striped bar represents fold change value obtained from NanoString nCounter XT platform. Concordance of directionality of fold change values between the two platforms confirm the RNA-Seq differential gene expression results.

\section{Discussion}

This manuscript presents the lymph node, spleen, and thymus transcriptome of wild Malaysian cynomolgus macaque sequenced with the Illumina HiSeq 2500 platform. A combined total of $174,208,078$ reads were obtained from the HiSeq 2500 sequencing run of the three tissues with three replicates each. Reference-guided mapping of the sequencing reads to the $M$. fascicularis reference genome revealed mapping percentages ranging from $53.00 \%$ to $63.00 \%$. To determine whether the low mapping percentage was caused by contaminants, the unmapped reads from M1, M2, and M5 Lymph were BLAST against NCBI's nucleotide (nt) database and classified into their specific taxa. The majority of the reads were found to map to Cercopithecidae and other primate families, while the remaining reads either did not have any BLASTN hits or were unassigned (Supplementary Data, Figure S16). This suggests the presence of novel transcripts within the lymph node, spleen, and thymus transcriptome that are yet to be described or annotated in the $M$. fascicularis reference genome, indirectly reflecting the incompleteness of the genome's annotations (Zhao \& Zhang, 2016).

Previous transcriptome sequencing endeavours via high-throughput sequencing methods were conducted on laboratory-bred cynomolgus macaques that originated from Vietnam, China, and Mauritius (Huh et al., 2012; Pipes et al., 2012; Peng et al., 2014). Our sequencing results show the expression of 253 spleen-specific genes, contrasting with Huh et al. (2012) whereby no spleen-specific genes were called in their sequencing endeavour. Such an occurrence is likely due to the lower total sequencing reads obtained in Huh et al.'s (2012) sequencing effort, amounting to 4 million reads across 16 different tissue libraries from two individuals compared with this study's 174.2 million reads across a total of nine tissue libraries from three individuals. An alternative explanation is that the study by Huh et al. (2012) contains 16 tissue samples, whereas the present study only contains 3 tissues, and is therefore easier to be tissue specific in the latter case. Peng et al. (2014) sequenced a total of 189.4 million reads from the lymph node, spleen, and thymus transcriptomes of Chinese cynomolgus macaque using the Illumina HiSeq 2000 technology. A preliminary comparison of gene expression values (normalised expression values) of 15 tissue-specific genes generated from this study with the gene expression values (normalised sFPKM) of Chinese cynomolgus macaques described in Peng et al. (2014) show 9 out of 15 gene expression patterns in concordance (Table S5), however the gene expression magnitude of certain genes such as $P R B 3$ and $Z G 16 B$ vary due possibly to variations in individuals and / or populations, which is evident in primate species (Enard et al., 2002; Hsieh et 
$634 a l ., 2003)$. In the future, more robust transcriptomic comparisons between the Malaysian 635 cynomolgus macaques and the Chinese and Mauritian cynomolgus macaques sequenced by 636 Pipes et al. (2012) and Peng et al. (2014) may potentially reveal more insights in the 637 transcriptomic and genetic differences between the three populations.

638

639

Concluding Remarks

640

641

642

643

644

645

646

647

648

649

650

651

652

653

654

655

656

657

658

659

660

661

662

663

Acknowledgements

664

665

666

667

668

669

670

In an immunological aspect, lymph node, spleen, and thymus transcriptomic data of wild Malaysian cynomolgus macaques generated from this study are potentially valuable for further studies. For AIDS-related immunology studies, the panel of genes expressed in the MHC I and MHC II pathways are advantageous for MHC genotyping studies of the Malaysian cynomolgus macaque population, which was suggested to have relatively higher nucleotide diversity compared with other populations of cynomolgus macaques in Southeast Asia (Smith, McDonough \& George, 2007). Additional screenings of MHC genotypes in Malaysian cynomolgus macaques may aid in further understanding and discovering novel immune regulation mechanisms and responses. Previous studies have characterised MHC genotypes as well as their effects on SIV progression in cynomolgus macaques (Burwitz et al., 2009; Campbell et al., 2009; Aarnink et al., 2011; Borsetti et al., 2012). The other benefit of transcriptomic data obtained from wild populations is how they more closely reflect the 'wild' and variable environment humans undergo on a daily basis. It is notable that in controlled laboratory environments, the absence of biotic and abiotic pressures associated with natural environments is unrepresentative of how immune responses function in the wild in response to pathogenic infections and injury. In assessing natural immune responses, both genetic and environmental factors play concerted roles. The transcriptomic information obtained from the three immune organs provides a fundamental outlook to the immune responses enriched in the wild cynomolgus macaque. Immunology data gleaned from wild cynomolgus macaque populations will benefit researchers in identifying significant immune responses and phenotypes that are translatable to natural human settings (Pedersen \& Babayan, 2011).

\section{Acknowledgements}

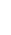
Malaysia, for the permission to conduct this study. We would also like to thank Dr. Ng Wei Lun (School of Life Sciences, Sun Yat-sen University), Nancy Liew Woan Charn (Laboratory of Vaccines and Immunotherapeutics, Institute of Bioscience, Universiti Putra Malaysia), and Mok Shao Feng for their invaluable guidance and assistance throughout the preparation of this manuscript.

671 
672 References

673

674 Aarnink A, Dereuddre-Bosquet N, Vaslin B, Le Grand R, Winterton P, Apoil PA, Blancher A.

675 2011. Influence of the MHC genotype on the progression of experimental SIV infection in the 676 Mauritian cynomolgus macaque. Immunogenetics 63:267-274 DOI 10.1007/s00251-010-0504-6.

677 Abdul-Latiff MAB, Ruslin F, Faiq H, Hairul MS, Rovie-Ryan JJ, Abdul-Patah P, Yaakop S, Md678 Zain BM. 2014. Continental Monophyly and Molecular Divergence of Peninsular Malaysia's 679 Macacafascicularisfascicularis. BioMed Research International2014:1-18 DOI $68010.1155 / 2014 / 897682$.

681 Abolins SR, Pocock MJ, Hafalla JC, Riley EM, Viney ME. 2011. Measures of immune function 682 of wild mice, Mus musculus. Molecular Ecology20:881-892 DOI: 10.1111/j.1365683 294X.2010.04910.x.

684 Alenquer M, Amorim MJ. 2015. Exosome biogenesis, regulation, and function in viral infection. 685 Viruses7:5066-5083 DOI 10.3390/v7092862.

686 Berry N, Ham C, Alden J, Clarke S, Stebbings R, Stott J, Ferguson D, Almond N. 2015. Live 687 attenuated simian immunodeficiency virus vaccination confers superinfection resistance against 688 macrophage-tropic and neurovirulent wild-type SIV challenge. Journal of General 689 Virology96:1918-1929 DOI 10.1099/vir.0.000135.

690 Blancher A, Bonhomme M, Crouau-Roy B, Terao K, Kitano T, Saitou N. 2008. Mitochondrial 691 DNA sequence phylogeny of 4 populations of the widely distributed cynomolgus macaque 692 (Macaca fascicularis fascicularis). Journal of Heredity 99:254-264 DOI 10.1093/jhered/esn003.

693 Borsetti A, Maggiorella MT, SernicolaL, Bellino S, Ferrantelli F, Belli R, Fulgenzi D, Mee ET, 694 Rose NJ, Cafaro A, Ensoli B, Titti F. 2012. Influence of MHC class I and II haplotypes on the 695 experimental infection of Mauritian cynomolgus macaques with SHIVSF162P4cy. Tissue 696 Antigens 80:36-45 DOI 10.1111/j.1399-0039.2012.01875.x.

697 Branchi V, Schaefer P, Semaan A, Kania A, Lingohr P, Kalff JC, Schäfer N, Kristiansen G, 698 Dietrich D,Matthaei H. 2016. Promoter hypermethylation of SHOX2 and SEPT9 is a potential 699 biomarker for minimally invasive diagnosis in adenocarcinomas of the biliary tract. Clinical 700 Epigenetics8:133 DOI 10.1186/s13148-016-0299-х.

701 Brendolan A, Rosado MM, Carsetti R, Selleri L, Dear TN. 2007. Development and function of 702 the mammalian spleen. Bioessays29:166-177DOI 10.1002/bies.20528.

703 Burwitz BJ, Pendley CJ, Greene JM, Detmer AM, Lhost JJ, Karl JA, Piaskowski SM,Rudersdorf 704 RA, Wallace LT, Bimber BN, Loffredo JT, Cox DG, Bardet W, Hildebrand W, Wiseman RW, 705 O'Connor SL, O'Connor DH. 2009. Mauritian cynomolgus macaques share two exceptionally 706 common major histocompatibility complex class I alleles that restrict simian immunodeficiency 707 virus-specific CD8+ T cells. Journal of Virology 83:6011-6019 DOI 10.1128/JVI.00199-09. 
708 Campbell KJ, Detmer AM, Karl JA, Wiseman RW,Blasky AJ, Hughes AL, Bimber BN, 709 O'Connor SL, O'Connor DH. 2009. Characterization of $47 \mathrm{MHC}$ class I sequences in Filipino 710 cynomolgus macaques. Immunogenetics 61:177-187 DOI 10.1007/s00251-008-0351-x.

711 Carlsson HE, Schapiro SJ, Farah I, Hau J. 2004. Use of primates in research: a global overview. 712 American Journal of Primatology 63:225-237 DOI 10.1002/ajp.20054.

713 Chen CC, Chen HY, Su KY, Hong QS, Yan BS, Chen CH, Pan SH, Chang YL, Hung PF, Yuan 714 S, Chang GC, Chen JJW, Yang PC, Yang YC, Yu SL. 2014. Shisa3 is associated with prolonged 715 survival through promoting $\beta$-catenin degradation in lung cancer. American Journal of 716 Respiratory and Critical Care Medicine 190:433-444 DOI 10.1164/rccm.201312-2256OC.

717 Czömpöly T, Lábadi Á, Kellermayer Z, Olasz K, Arnold HH, Balogh P. 2011. Transcription 718 factor Nkx2-3 controls the vascular identity and lymphocyte homing in the spleen. The Journal 719 of Immunology 186:6981-6989 DOI 10.4049/jimmunol.1003770.

720 Ebeling M, Kung E, See A, Broger C, Steiner G, Berrera M, Heckel T, Iniguez L, Albert T, 721 Schmucki R, Biller H, Singer T, Certa U. 2011. Genome-based analysis of the nonhuman 722 primate Macacafascicularis as a model for drug safety assessment. Genome Research 21:17467231756 DOI 10.1101/gr.123117.111.

724 Enard W, Khaitovich P, Klose J, Zöllner S, Heissig F, Giavalisco P, Nieselt-Struwe K, 725 Muchmore E, Varki A, Ravid R,Doxiadis GM, Bontrop RE, Pääbo S. 2002. Intra-and interspecific variation in primate gene expression patterns. Science 296:340-343 DOI 10.1126/science.1068996.

Faissner A, Heck N, Dobbertin A, Garwood J. 2006. DSD-1-Proteoglycan/Phosphacan and receptor protein tyrosine phosphatase-beta isoforms during development and regeneration of neural tissues. In:Bähr M, ed. Brain Repair. New York: Springer Science+Business Media, 2553 DOI 10.1007/0-387-30128-3_3.

732 Foletta VC, Segal DH, Cohen DR. 1998. Transcriptional regulation in the immune system: all 733 roads lead to AP-1. Journal of Leukocyte Biology63:139-152

734 Frick VO, Rubie C, Keilholz U, Ghadjar P. 2016. Chemokine/chemokine receptor pair 735 CCL20/CCR6 in human colorectal malignancy: An overview. World Journal of 736 Gastroenterology22:833-841 DOI 10.3748/wjg.v22.i2.833.

737 Gillece-Castro BL, Prakobphol A, Burlingame AL, Leffler H, Fisher SJ. 1991. Structure and 738 bacterial receptor activity of a human salivary proline-rich glycoprotein. Journal of Biological 739 Chemistry266:17358-17368

740 Gu S, Wei N, Yu L, Fei J, Chen Y. 2008. Shox2-deficiency leads to dysplasia and ankylosis of 741 the temporomandibular joint in mice. Mechanisms of Development 125:729-742 DOI 742 10.1016/j.mod.2008.04.003. 
743 Han Y, Gao S, Muegge K, Zhang W, Zhou B. 2015. Advanced applications of RNA sequencing

744 and challenges. Bioinformatics and Biology Insights 9(Suppl 1):29-46 DOI

745 10.4137/BBI.S28991.

746 Haus T, Ferguson B, Rogers J, Doxiadis G, Certa U, Rose NJ, Teepe R, Weinbauer GF, Roos C.

747 2014. Genome typing of nonhuman primate models: implications for biomedical research.

748 Trends in Genetics30:482-487 DOI 10.1016/j.tig.2014.05.004.

749 Herring CA, Singer CM, Ermakova EA, Khairutdinov BI, Zuev YF, Jacobs DJ, Nesmelova IV.

750 2015. Dynamics and thermodynamic properties of CXCL7 chemokine. Proteins: Structure,

751 Function, and Bioinformatics83:1987-2007 DOI 10.1002/prot.24913.

752 Higashino A, Sakate R, Kameoka Y, Takahashi I, Hirata M, Tanuma R, Masui T, Yasutomi Y, 753 Osada N. 2012. Whole-genome sequencing and analysis of the Malaysian cynomolgus macaque 754 (Macacafascicularis) genome. Genome Biology 13:R58 DOI 10.1186/gb-2012-13-7-r58.

755 Higgs S,Ziegler SA. 2010. A nonhuman primate model of chikungunya disease. The Journal of 756 Clinical Investigation 120:657-660 DOI 10.1172/JCI42392.

757 Hsieh WP, Chu TM, Wolfinger RD, Gibson G. 2003. Mixed-model reanalysis of primate data 758 suggests tissue and species biases in oligonucleotide-based gene expression 759 profiles. Genetics 165:747-757.

760 Hu D, Zhou W, Wang F, Shu SM, Fan LL, He J, Wang P, He YL, Du W, Zhang JH, Duan JX, 761 Sun L, Zheng J, Li XQ, Li HY, Feng XL, Huang SA. 2016. Development of a NanoString assay 762 to detect leukemogenic fusion transcripts in acute myeloid leukemia. International Journal of 763 Laboratory Hematology6:663-673 DOI 10.1111/ijlh.12555.

764 Huh JW, Kim YH, Park SJ, Kim DS, Lee SR, Kim KM, Jung KJ, Kim JS, Song BS, Sim BW, 765 Kim SU, Kim SH, Chang KT. 2012. Large-scale transcriptome sequencing and gene analyses in 766 the crab-eating macaque (Macacafascicularis) for biomedical research. BMC Genomics 13:163 767 DOI 10.1186/1471-2164-13-163.

768 Jia H, Wang X, Liu F, Guenther UP, Srinivasan S, Anderson JT, Jankowsky E. 2011. The RNA 769 helicase Mtr4p modulates polyadenylation in the TRAMP complex. Cell145:890-901 DOI 770 10.1016/j.cell.2011.05.010.

771 Kappelmann M, Bosserhoff A, Kuphal S. 2014. AP-1/c-Jun transcription factors: regulation and 772 function in malignant melanoma. European Journal of Cell Biology93:76-81 DOI 773 10.1016/j.ejcb.2013.10.003.

774 Lee A, Khiabanian H, Kugelman J, Elliott O, Nagle E, Yu GY, Warren T, Palacios G, Rabadan 775 R. 2014a. Transcriptome reconstruction and annotation of cynomolgus and African green 776 monkey. BMC Genomics 15:846 DOI 10.1186/1471-2164-15-846.

777 Lee Y, Kim YH, Park SJ, Huh JW, Kim SH, Kim SU, Kim JS, Jeong KJ, Lee KM, Hong Y, Lee 778 SR,Chang KT. 2014b. Insullin/IGF signaling-related gene expression in the brain of a sporadic 
779

780

781

782

783

784

785

786

787

788

789

790

791

792

793

794

795

796

797

798

799

800

801

802

803

804

805

806

807

808

809

810

811

812

813

814

815

Alzheimer's disease monkey model induced by intracerebroventricular injection of streptozotocin. Journal of Alzheimer's Disease38:251-267 DOI 10.3233/JAD-130776.

Liedigk R, Kolleck J, Böker KO, Meijaard E, Md-Zain BM, Abdul-Latiff MAB, Ampeng A, Lakim M, Abdul-Patah B, Tosi AJ, Brameier M, Zinner D, Roos C. 2015. Mitogenomic phylogeny of the common long-tailed macaque (Macacafascicularisfascicularis). BMC Genomics 16:222DOI 10.1186/s12864-015-1437-0.

Liu W, Tang L, Zhang G, Wei H, Cui Y, Guo L, Gou Z, Chen X, Jiang D, Zhu Y, Kang G,He F. 2004. Characterization of a novel C-type lectin-like gene, LSECtin demonstration of carbohydrate binding and expression in sinusoidal endothelial cells of liver and lymph node. Journal of Biological Chemistry279:18748-18758 DOI 10.1074/jbc.M311227200.

Molleston JM, Sabin LR, Moy RH, Menghani SV, Rausch K, Gordesky-Gold B, Hopkins KC, Zhou R, Jensen TH, Wilusz JE,Cherry S. 2016. A conserved virus-induced cytoplasmic TRAMP-like complex recruits the exosome to target viral RNA for degradation. Genes \& Development30:1658-1670 DOI 10.1101/gad.284604.116.

Nauseef WM, McCormick SJ, Clark RA. 1995. Calreticulin functions as a molecular chaperone in the biosynthesis of myeloperoxidase. Journal of Biological Chemistry270:4741-4747 DOI 10.1074/jbc.270.9.4741.

Nufer O, Kappeler F, Guldbrandsen S, Hauri HP. 2003. ER export of ERGIC-53 is controlled by cooperation of targeting determinants in all three of its domains. Journal of Cell Science 116:4429-4440 DOI 10.1242/jcs.00759.

Nunamaker EA, Halliday LC, Moody DE, Fang WB, Lindeblad M, Fortman JD. 2013. Pharmacokinetics of 2 formulations of buprenorphine in macaques (Macacamulatta and Macacafascicularis). Journal of the American Association for Laboratory Animal Science 52:4856.

Oppenheim FG, Salih E, Siqueira WL, Zhang W, Helmerhorst EJ. 2007. Salivary proteome and its genetic polymorphisms. Annals of the New York Academy of Sciences 1098:22-50 DOI 10.1196/annals.1384.030.

Osada N, Hettiarachchi N, Babarinde IA, Saitou N, Blancher A. 2015. Whole-genome sequencing of six Mauritian cynomolgus macaques (Macacafascicularis) reveals a genome-wide pattern of polymorphisms under extreme population bottleneck. Genome Biology and Evolution7:821-830 DOI 10.1093/gbe/evv033.

Panos M, Christophi GP, Rodriguez M, Scarisbrick IA. 2014. Differential expression of multiple kallikreins in a viral model of multiple sclerosis points to unique roles in the innate and adaptive immune response. Biological Chemistry395:1063-1073 DOI 10.1515/hsz-2014-0141.

Park HD, Lee Y, Oh YK, Jung JG, Park YW, Myung K, Kim KH, Koh SS, Lim DS. 2011. Pancreatic adenocarcinoma upregulated factor promotes metastasis by regulating TLR/CXCR4 activation. Oncogene30:201-211 DOI 10.1038/onc.2010.401. 
816 Patel K, Jhamb SS, Singh PP. 2011. Models of latent tuberculosis: their salient features, 817 limitations, and development. Journal of Laboratory Physicians3:75-79 DOI 10.4103/09748182727.86837.

819 Pedersen AB, Babayan SA. 2011. Wild immunology. Molecular Ecology20:872-880 DOI $82010.1111 /$ j.1365-294X.2010.04938.x.

821 Peng X, Thierry-Mieg J, Thierry-Mieg D, Nishida A, Pipes L, Bozinoski M, Thomas MJ, Kelly 822 S, Weiss JM, Raveendran M, Muzny D, Gibbs RA, Rogers J, Schroth GP, Katze MG, Mason 823 CE. 2014. Tissue-specific transcriptome sequencing analysis expands the non-human primate 824 reference transcriptome resource (NHPRTR). Nucleic Acids Research43:D737-D742 DOI 825 10.1093/nar/gku1110.

826 Perelman P, Johnson WE, Roos C, Seuanez HN, Horvath JE, Moreira MA, Kessing B, Pontius J, 827 Roelke M, Rumpler Y, Schneider MPC, Silva A, O’Brien SJ, Pecon-Slattery J. 2011. A 828 molecular phylogeny of living primates. PLoS Genetics DOI 10.1371/journal.pgen.1001342.

829 Pipes L, Li S, Bozinoski M, Palermo R, Peng X, Blood P, Kelly S, Weiss JM, Thierry-Mieg J, 830 Thierry-Mieg D, Zumbo P, Chen R, Schroth GP, Mason CE, Katze MG. 2013. The non-human 831 primate reference transcriptome resource (NHPRTR) for comparative functional genomics. 832 Nucleic Acids Research41:D906-D914 DOI 10.1093/nar/gks1268.

833 Radke L, Giese C, Lubitz A, Hinderlich S, Sandig G, Hummel M, Frohme M. 2014. Reference 834 gene stability in peripheral blood mononuclear cells determined by qPCR and NanoString. 835 MicrochimicaActa181:1733-1742 DOI 10.1007/s00604-014-1221-x.

836 Rovie-Ryan JJ, Abdullah MT, Sitam FT, Abidin ZZ, Tan SG. 2013. Y-chromosomal gene flow 837 of Macacafascicularis (Cercopithecidae) between the insular and mainland peninsula of Penang 838 state, Malaysia. Journal of Science and Technolology in the Tropics9:113-126.

839 Schmid M,Jensen TH. 2008. The exosome: a multipurpose RNA-decay machine. Trends in 840 Biochemical Sciences33:501-510 DOI 10.1016/j.tibs.2008.07.003.

841 Shively CA, Register TC, Appt SE, Clarkson TB. 2015. Effects of Long Term Sertraline 842 Treatment and Depression on Coronary Artery Atherosclerosis in Premenopausal Female 843 Primates. Psychosomatic Medicine 77:267-278 DOI 10.1097/PSY.0000000000000163.

844 Smith DG, McDonough JW, George DA. 2007. Mitochondrial DNA variation within and among 845 regional populations of longtail macaques (Macacafascicularis) in relation to other species of the 846 fascicularis group of macaques. American Journal of Primatology69:182-198 DOI 847 10.1002/ajp.20337.

848 Staals RHJ, Bronkhorst AW, Schilders G, Slomovic S, Schuster G, Heck AJR, Raijmakers R, 849 Pruijn GJM. 2010. Dis3-like 1: a novel exoribonuclease associated with the human exosome. The 850 EMBO Journal29:2358-2367DOI 10.1038/emboj.2010.122. 
851 Tosi AJ, Coke CS. 2007. Comparative phylogenetics offer new insights into the biogeographic 852 history of Macacafascicularis and the origin of the Mauritian macaques. Molecular 853 Phylogenetics and Evolution42:498-504 DOI 10.1016/j.ympev.2006.08.002.

854 Trichel AM, Rajakumar PA, Murphey-Corb M. 2002. Species-specific variation in SIV disease 855 progression between Chinese and Indian subspecies of rhesus macaque. Journal of Medical 856 Primatology31:171-178 DOI 10.1034/j.1600-0684.2002.02003.x.

857 Wang C, Li N, Liu X, Zheng Y, Cao X. 2008. A novel endogenous human CaMKII inhibitory 858 protein suppresses tumor growth by inducing cell cycle arrest via p27 stabilization. Journal of 859 Biological Chemistry283:11565-11574 DOI 10.1074/jbc.M800436200.

860 Wang Y, Tang X, Yu B, Gu Y, Yuan Y, Yao D, Ding F, Gu X. 2012. Gene network revealed 861 involvements of Birc2, Birc3 and Tnfrsfla in anti-apoptosis of injured peripheral nerves. PLoS 862 One7:e43436 DOI 10.1371/journal.pone.0043436.

863 Watson P, Townley AK, Koka P, Palmer KJ, Stephens DJ. 2006. Sec16 defines endoplasmic 864 reticulum exit sites and is required for secretory cargo export in mammalian cells. Traffic 7:1678865 1687 DOI 10.1111/j.1600-0854.2006.00493.x.

866 Weissman JT, Plutner H,Balch WE. 2001. The mammalian guanine nucleotide exchange factor $867 \mathrm{mSec} 12$ is essential for activation of the Sar1 GTPase directing endoplasmic reticulum export. 868 Traffic 2:465-475 DOI 10.1034/j.1600-0854.2001.20704.x.

869 Yan G, Zhang G, Fang X, Zhang Y, Li C, Ling F, Cooper DN, Li Q, Li Y, van Gool AJ, Du H, 870 Chen J, Chen R, Zhang P, Huang Z, Thompson JR, Meng Y, Bai Y, Wang J, Zhuo M, Wang T, 871 Huang Y, Wei L, Li J, Wang Z. 2011. Genome sequencing and comparison of nonhuman 872 primate animals models, the cynomolgus and Chinese rhesus macaques. Nature 873 Biotechnology 29:1019-1023 DOI 10.1038/nbt.1992.

874 Zhang X, Jiang G, Cai Y, Monkley SJ, Critchley DR, Sheetz MP. 2008. Talin depletion reveals 875 independence of initial cell spreading from integrin activation and traction. Nature Cell 876 Biology 10:1062-1068 DOI 10.1038/ncb1765.

877 Zhao S,Zhang B. 2016. Impact of Gene Annotation on RNA-seq Data Analysis. In: Kulski J, 878 ed.Next Generation Sequencing - Advances, Applications and Challenges.InTech, DOI $87910.5772 / 61197$. 